\title{
Steroidresistenz bei organisierender Pneumonie bedingt durch Lungenkryptokokkose
}

\author{
Motoko Nomura $^{\mathrm{a}}$ Hiromitsu Ohta ${ }^{\mathrm{a}}$ Masahiro Hiruta $^{\mathrm{b}}$ Fumiaki Kudo $^{\mathrm{a}}$ \\ Fumiyoshi Ohyanagi $^{a}$ Yasuhiro Yamaguchia
}

${ }^{a}$ Abteilung für Atemwegsmedizin, Jichi Medical University Saitama Medical Center, Saitama, Japan;

${ }^{\mathrm{b}}$ Abteilung für Pathologie, Jichi Medical University Saitama Medical Center, Saitama, Japan

\begin{abstract}
Schlüsselwörter
Kryptokokkose $\cdot$ kryptogene organisierende Pneumonie .

immungeschwächt · transbronchiale Lungenbiopsie
\end{abstract}

\section{Zusammenfassung}

Die kryptogene organisierende Pneumonie (COP) spricht in der Regel gut auf eine Steroidtherapie an. Bei einem Ausschleichen der Steroiddosis kommt es jedoch häufig zu Rezidiven. In unserem Krankenhaus stellte sich ein 74-jähriger Mann mit Verdacht auf steroidresistente COP vor. Eine Thorax-Computertomographie (CT) ergab trotz Verabreichung oraler Steroide in mittlerer Dosierung neue Konsolidierungen im Bereich des linken Lungenunterlappens. Bei einer erneuten transbronchialen Lungenbiopsie wurde eine Lungenkryptokokkose festgestellt. Nach Einleitung einer Be- handlung mit Antimykotika verkleinerten sich die Konsolidierungen im linken Unterlappen sukzessive. Bei immungeschwächten Patienten mit Lungenkryptokokkose kommt es zu unterschiedlichen CT-Befunden, wobei Konsolidierungen häufig beobachtet werden. Im Falle einer steroidrefraktären COP sollte eine zugrunde liegende Lungenkryptokokkose in Betracht gezogen werden.

(c) 2020 Die Autoren. Respirology Case Reports veröffentlicht durch John Wiley \& Sons Australia, Ltd im Auftrag von The Asian Pacific Society of Respirology

\section{Einführung}

Die Kryptokokkose ist eine häufige Infektion mit dem Sprosspilz Cryptococcus neoformans, einer bekapselten runden Hefe. Der Pilz ist weltweit verbreitet und findet sich in mit Taubenkot kontaminierten Erdböden, verrottendem Holz und anderem organischen Material. Der Mensch infiziert sich durch Inhalation von Kryptokokkenpartikeln. Als Erstmanifestation der Infektion entwickelt sich eine Pneumonie mit anschließender Ausbildung von Granulomen. Zu einer Lungenkryptokokkose kommt es sowohl bei immunkompetenten als auch bei immunsupprimierten Wirten.

Bei der organisierenden Pneumonie (OP) handelt es sich vermutlich um einen entzündlichen Reparaturvorgang, der nach einer
Schädigung des Alveolarepithels ausgelöst wird. Eine sekundäre OP kann durch eine konkrete Ursache bedingt sein, beispielsweise eine Infektion, eine Bindegewebskrankheit, verschiedene Arzneimittel, Strahlung, Malignität und andere Formen einer interstitiellen Pneumonie. Lässt sich keine Ursache identifizieren, erfüllt die OP die Definition einer kryptogenen OP (cryptogenic organizing pneumonia, COP). Bei der Computertomographie (CT) zeigen sich als charakteristische Manifestationen der COP beidseitige, ungleichmäßige oder diffuse Konsolidierungen oder Milchglastrübungen [1].

Die COP bessert sich in der Regel unter Steroidtherapie deutlich, beim Ausschleichen der Steroiddosis kann es jedoch zu einem Rezidiv kommen. Laut Lazor et al. kam es bei 58\% der Patienten mit mittels Biopsie nachgewiesener COP zu einem oder mehr Re- (c) 2020 The Author(s)

Published by S.Karger GmbH, Freiburg

This article is licensed under the Creative Commons Attribution 4.0 International License (CC BY) (http://www.karger.com/Services/Open AccessLicense). Usage, derivative works and distribution are permitted provided that proper credit is given to the author and the original publisher.
Hiromitsu Ohta

Department of Respiratory Medicine

Jichi Medical University Saitama Medical Center

Amanuma 1-847, Oomiya, Saitama 330-0834, Japan

hootatky@jichi.ac.jp 

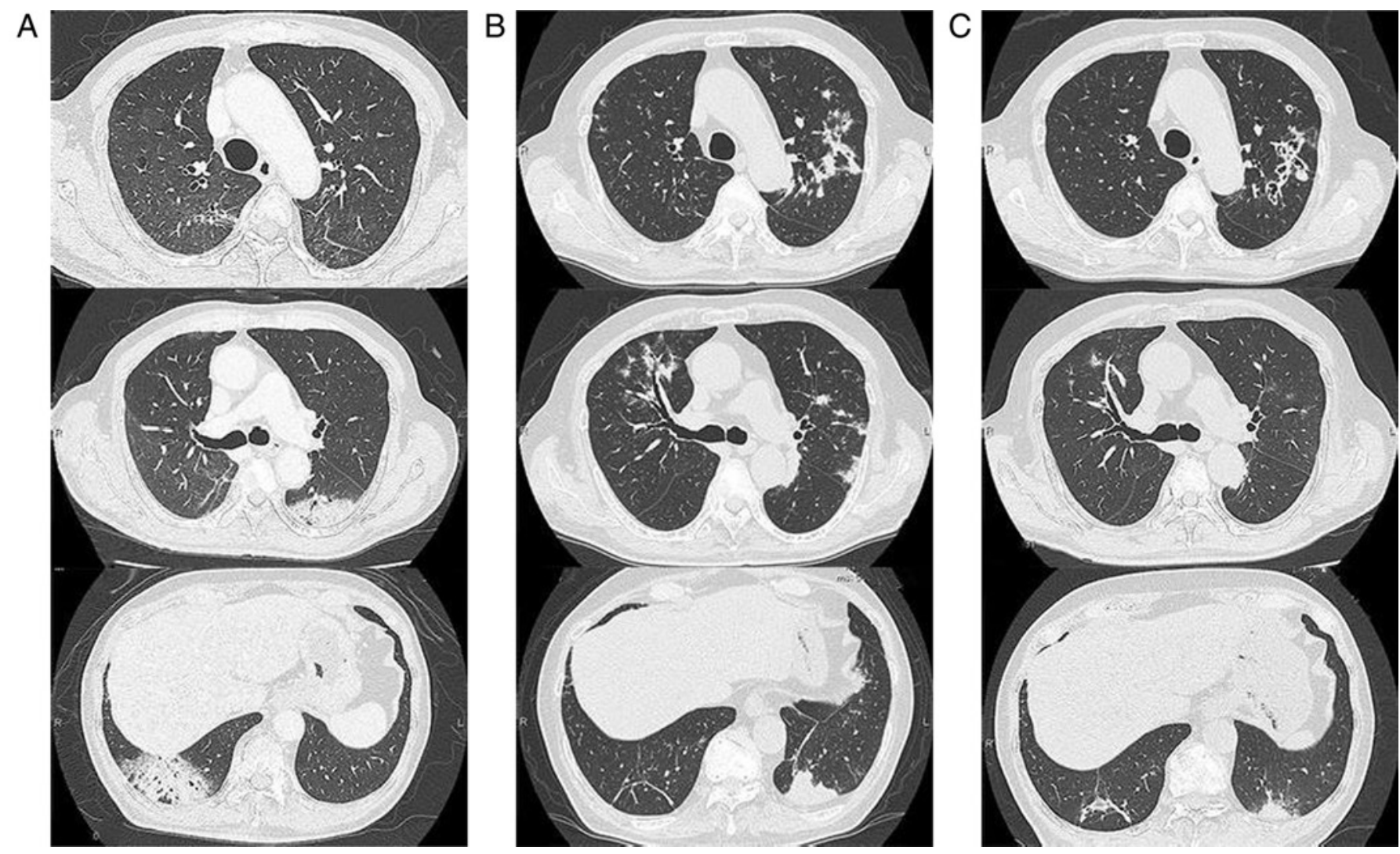

Abb. 1. Radiologische Befunde. (a) Thorax-Computertomographie (CT)-Aufnahme zum Zeitpunkt der Initialdiagnose einer kryptogenen organisierenden Pneumonie (COP). Der Patient hatte noch kein Kortikosteroid erhalten. Auf mehreren Lungenlappen sind Konsolidierungen zu erkennen. In der Lunge zeigten sich keine nodulären Verschattungen. (b) Die beim ersten Termin in unserem Krankenhaus angefertigte Thorax-CT-Aufnahme zeigt eine Konsolidierung des linken Unterlappens und mehrere noduläre Verschattungen mit verdickten Bronchialwänden in beiden Lungenflügeln. Die initialen Konsolidierungen, die be Diagnose der COP beobachtet worden waren, lagen nicht mehr vor. (c) Thorax-CT-Aufnahme nach sechsmonatiger Behandlung mit Fluconazol. Die Konsolidierungen des linken Unterlappens waren kleiner geworden, und die große Zahl nodulärer Verschattungen hatte sich reduziert. Im linken Oberlappen ist eine Bronchiektase erkennbar.

zidiven, wobei das erste Rezidiv bei 68\% dieser Patienten unter der initialen Steroidtherapie auftrat. Die meisten Patienten, bei denen ein Rezidiv auftrat, erhielten zu diesem Zeitpunkt weniger als 10 mg/Tag Prednisolon (PSL), und bei Patienten unter hoch dosiertem PSL (> 20 mg/Tag) traten Rezidive selten auf [2]. Bei manchen Infektionskrankheiten kommt es unter einer Steroidtherapie häufig zu atypischen klinischen und radiologischen Manifestationen. Aus diesem Grunde sind refraktäre COP-Rezidive sorgfältig erneut zu beurteilen, hauptsächlich dann, wenn das Rezidiv unter hoch dosiertem PSL auftritt. Wir berichten über den Fall eines immunkompetenten Patienten mit bronchoskopisch diagnostizierter COP, der auf eine mittlere Steroiddosis nicht mehr ansprach. Auf Grundlage einer erneuten Bronchoskopie wurde bei ihm letztlich die Diagnose Lungenkryptokokkose gestellt.

\section{Fallbericht}

Ein 74-jähriger Mann stellte sich in einer örtlichen Poliklinik mit seit 2 Monaten bestehenden Beschwerden im Brustkorb und Atemnot vor. Seine Symptome hatten sich unter einer Antibiose nicht gebessert. Das Thorax-Röntgen und eine CT zeigten beid- seitig multiple Konsolidierungen in den Lungenfeldern (Abb. 1a). Eine Analyse der bronchoalveolären Lavage-Flüssigkeit (BALF) ergab eine erhöhte Lymphozytenzahl (34\%). Eine BALF-Kultur ergab keine Mikroorganismen. Mit Hilfe einer transbronchialen Lungenbiopsie (TBLB) aus dem rechten Lungenunterlappen gewonnene Proben zeigten eine erhaltene Grundarchitektur sowie eine Ansammlung alveolärer Makrophagen und einige wenige Fibroblasten in Alveolarräumen nahe der Bronchiole, auch wenn keine intraluminale organisierende Fibrose innerhalb von Alveolen und Bronchiolen nachgewiesen wurde (Abb. 2a, b). Eine Gram-Färbung, eine Anfärbung zum Nachweis säurefester Bakterien sowie eine Grocott-Färbung des Lungengewebes waren negativ (Abb. 2c). Auf der Grundlage dieser Ergebnisse erhielt der Patient die Diagnose COP und wurde mit einem oralen Kortikosteroid, Prednisolon (PSL) $40 \mathrm{mg} / \mathrm{Tag}(0,75 \mathrm{mg} / \mathrm{kg} / \mathrm{Tag})$, behandelt. Einen Monat später hatten sich seine Symptome und seine Thorax-Radiologiebefunde verbessert. Daraufhin wurde die Kortikosteroiddosis schrittweise reduziert. Bei Erreichen einer PSL-Dosis von $10 \mathrm{mg} /$ Tag zeigte sich im Thorax-Röntgen und -CT eine Exazerbation der beidseitigen Konsolidierung. Nach Erhöhung der PSL-Dosis auf $40 \mathrm{mg} /$ Tag besserte sich die Mehrzahl 
B
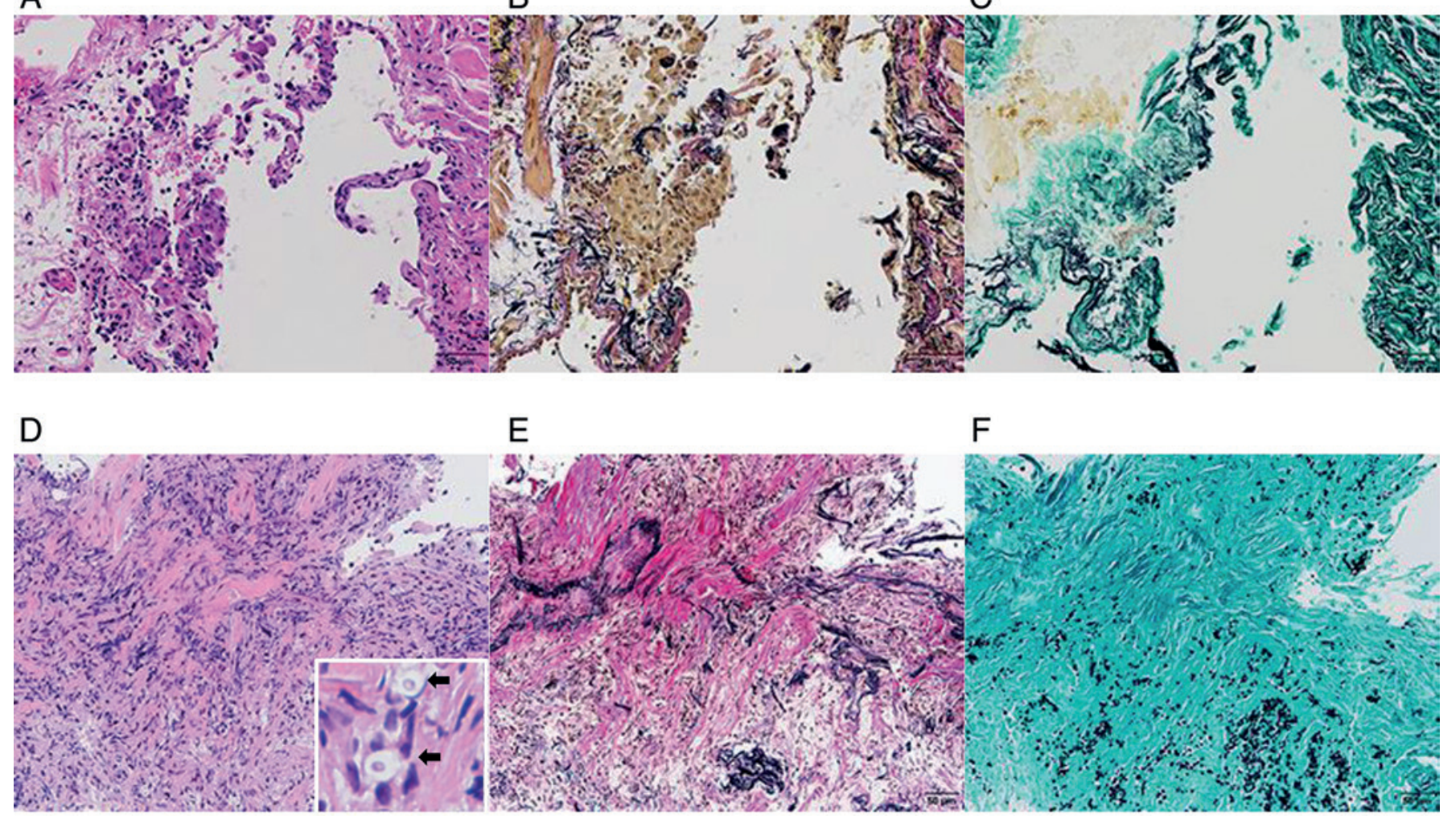

Abb. 2. Transbronchiale Biopsie-Proben. (a) Histopathologische Untersuchung der transbronchialen Biopsie-Proben aus dem rechten Unterlappen (durchgeführt im ersten Krankenhaus vor Einleitung der Kortikosteroidbehandlung; Hämatoxylin-Eosin-Färbung, 20x). (b) Eine Elastika-Färbung nach van Gieson (EVG) derselben Probe wie in (a) zeigt eine Ansammlung alveolärer Makrophagen und einige wenige Fibroblasten in den Alveolarräumen nahe der Bronchiole. (c) Grocott-Färbung derselben Probe wie in (a). In dem Gewebe sind keine Hefe- oder Bakterienzellen erkennbar. (d) Histopathologische Untersuchung der transbronchialen Biopsie-Proben aus dem linken Unterlappen, durchgeführt in unserem Krankenhaus (Hämatoxylin-Eosin-Färbung, 10x). (e) EVG-Färbung derselben Probe wie in (d). Im Interstitium und in den Alveolarräumen ist eine Vielzahl von Hefen und Makrophagen zu erkennen. Beachtenswert sind die kleinen runden Sphären im Zytoplasma der Makrophagen (Pfeile in der vergrößerten Ansicht im Quadrat). (f) Die Grocott-Färbung derselben Probe wie in (d) zeigt eine Vielzahl von Hefen im Interstitium und Alveolarraum.

der Konsolidierungen erneut. Als die Dosis auf 15 mg/Tag gesenkt wurde, zeigten sich im Thorax-Röntgen und -CT allerdings neue Konsolidierungen im linken Unterlappen. Die PSL-Dosis wurde erneut auf $40 \mathrm{mg} / \mathrm{Tag}$ erhöht, und der Patient wurde an unser Krankenhaus überwiesen.

Die Vitalzeichen des Patienten waren bei seinem ersten Termin in unserem Krankenhaus unauffällig, auch bei der körperlichen Untersuchung wurden bis auf ein peripheres Taubheitsgefühl in beiden Füßen keine Anomalien festgestellt. Der Patient war wegen einer Spinalkanalstenose, Hypertonie und Hyperurikämie vorbehandelt worden, ansonsten aber gesund. Er war als Landwirt in der Produktion von Salatgurken tätig und konstant Düngemitteln auf Hühnerkotbasis ausgesetzt. Die labortechnischen Untersuchungen in Bezug auf die Zahl der Blutzellen und biochemischen Parameter waren ohne Befund. Ein Thorax-Röntgen zeigte infiltrative Verschattungen im linken Lungenfeld, und eine Thorax-CT ergab Konsolidierungen des linken Unterlappens sowie beidseitig multiple ungleichmäßige Verschattungen mit verdickten Bronchialwänden (Abb. 1b). Es wurde eine weitere Bronchoskopie durchgeführt, und die BALF aus der Lingula pulmonis sinistri zeigte eine erhöhte Lymphozytenzahl (20,1\%). Die histopa- thologische Untersuchung des bei der TBLB des linken Unterlappens gewonnenen Biopsats ergab Hefen in großer Zahl und eine Infiltration von Makrophagen in den Alveolarraum. Einige runde Hefen wurden durch Makrophagen phagozytiert (Abb. 2d - f). Die BALF-Kultur war negativ. Sein KryptokokkenAntigen-Serumtiter war positiv und betrug 1:2048, und er testete negativ auf $\beta$-D-Glukan im Serum. Aufgrund seiner starken Lendenwirbelsäulenverkrümmung konnten keine Proben durch Lumbalpunktion genommen werden. Eine kontrastverstärkte Magnetresonanztomographie ergab keine Hinweise auf eine Meningitis, und er zeigte auch keine entsprechenden klinischen Symptome. Daher wurde bei dem Patienten eine Lungenkryptokokkose ohne Meningitis diagnostiziert. Es wurde bei ihm eine Behandlung mit Fluconazol 400 mg/Tag eingeleitet, und die PSLDosis wurde schrittweise reduziert. Nach sechs Monaten war die PSL-Dosis auf $5 \mathrm{mg} /$ Tag gesenkt worden. Wie die Thorax-CT ergab, waren die Konsolidierungen der linken Lunge stetig geschrumpft. Die Mehrzahl der nodulären Verschattungen war verschwunden, was zu einer Dilatation der Bronchien führte (Abb. 1c). 

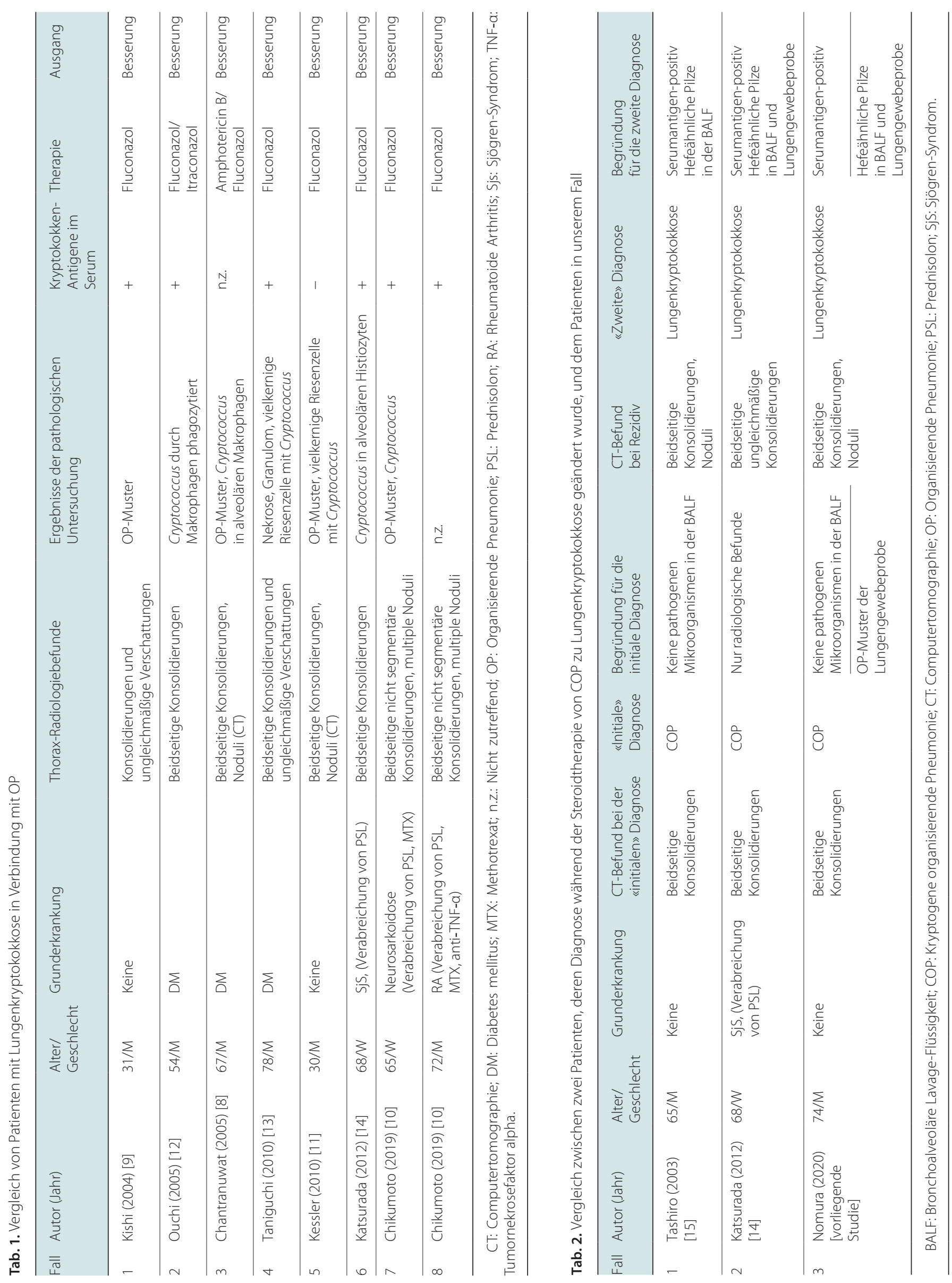


\section{Diskussion}

Die häufigsten radiologischen Befunde bei einem immunkompetenten Patienten mit Lungenkryptokokkose sind Noduli und Raumforderungen mit oder ohne Kavitation [3, 4]. Die meisten Patienten, die Konsolidierungen aufweisen, zeigen ebenfalls Noduli oder Kavitäten. Bei immunkompetenten Patienten aktiviert Cryptococcus die Th1-Antwort. Lymphozyten und Makrophagen umgeben das Cryptococcus und bilden Granulome, die auf dem Thorax-CT als Noduli erkennbar sind. Murayama et al. analysierten hoch auflösende CT-Aufnahmen von 13 immunkompetenten $\mathrm{Pa}$ tienten mit Lungenkryptokokkose [3]. Sie stellten fest, dass sich die Hauptmanifestationen in zwei Erscheinungsformen unterteilen lassen: multiple Noduli $(n=7)$ und ein einzelner Nodulus $(n=6)$. In zwei Fällen beobachteten sie ein Konsolidierungsmuster mit multiplen Noduli.

Immungeschwächte Patienten mit Lungenkryptokokkose zeigen verschiedene CT-Befunde. Bei diesen Patienten wird die Th1-Antwort nicht so stark aktiviert, dass sich ein Granulom bildet, und meist wandern Pilze in die umgebenden Gewebe ein. Zu Konsolidierungen kommt es bei immungeschwächten Patienten häufiger als bei immunkompetenten Patienten [5, 6]. Yanagawa et al. verglichen die CT-Befunde von Patienten mit Lungenkryptokokkose unter PSL-Behandlung in drei Gruppen: immunkompetente Patienten, AIDS-Patienten bzw. Patienten mit rheumatoider Arthritis (RA) [5]. Der häufigste Befund in allen Gruppen waren Noduli. Konsolidierungen und Milchglastrübungen fanden sich bei $30 \%$ der Patienten in der AIDS- und RA-Gruppe, jedoch nicht in der Gruppe immunkompetenter Patienten.

Mehrere Fallberichte zu Lungenkryptokokkose stellen die radiologischen und pathologischen Befunde bei OP vor (Tab. 1) [7-14]. Die meisten der Patienten waren immungeschwächt und zeigten im Thorax-CT beidseitig Konsolidierungen. Allerdings zeigten sich auch bei zwei immunkompetenten Patienten Konsolidierungen im Thorax-CT. In drei Fällen sprachen die Patienten nicht auf Steroide oder Immunsuppressiva an. Alle Patienten wurden mit einem Antimykotikum erfolgreich behandelt.

Es liegen zwei Fallberichte von Patienten vor, bei denen ursprünglich COP diagnostiziert und dann unter der Steroidtherapie die Diagnose Lungenkryptokokkose gestellt wurde (Tab. 2) [14, 15]. In einem Fall kamen Katsurada et al. zu dem Schluss, dass die Lungenkryptokokkose fälschlicherweise als COP diagnostiziert wurde, da keine pathologische Untersuchung durchgeführt wurde [14]. In dem anderen Fall gingen Tashiro et al. nach Verabreichung einer Steroidbehandlung bei dem Patienten mit COP von einer opportunistischen Kryptokokkeninfektion aus, da die erste pathologische und bakterielle Untersuchung mittels Bronchoskopie keine Hinweise auf eine Kryptokokkeninfektion ergeben hatte und sich die initiale Konsolidierung lediglich unter der Steroidtherapie besserte [15]. Es ist von wesentlicher Bedeutung, die COP von einer sekundären OP abzugrenzen, da zur Behandlung der sekundären OP häufig eine Therapie der Grunderkrankung erforderlich ist. Allerdings ist eine Unterscheidung zwischen sekundärer OP und COP in der klinischen Praxis nicht einfach. Drakopanagiotakis et al. haben kürz- lich berichtet, dass die klinischen und radiologischen Befunde von Patienten mit COP bzw. sekundärer OP ähnlich und nicht spezifisch sind [16].

Serumantigen-Tests auf Kryptokokkose können bei der Differenzierung zwischen Lungenkryptokokkose und COP hilfreich sein. Die Empfindlichkeit und die Spezifität von Serumantigen-Tests wurden anhand von 195 Serumproben von 25 Patienten mit Lungenkryptokokkose und 170 Patienten ohne Kryptokokkose untersucht. Ein Grenzwert von $\geq 1: 8$ zeigte eine Empfindlichkeit von $76 \%$ (19/25) und eine Spezifität von 98,9\% (168/170) [17]. In unserer Übersichtsarbeit wurde der Serumantigen-Test auf Kryptokokkose bei sieben Patienten mit Lungenkryptokokkose und einem OPMuster im Thorax-CT überprüft, und bei sechs von ihnen fiel der Test positiv aus (Tab. 2).

Unserer Vermutung nach ist der Umstand, dass unser Patient mit COP eine sekundäre Lungenkryptokokkose entwickelte, hauptsächlich auf drei Gründe zurück zu führen. Zum ersten ergab die initiale radiologische Untersuchung des Patienten multiple Konsolidierungen ohne Noduli, was für einen immunkompetenten Patienten mit Lungenkryptokokkose einen seltenen Thorax-CT-Befund darstellt. Zum zweiten sprachen die Konsolidierungen auf der Thorax-CT-Aufnahme, die zum Zeitpunkt der COP-Diagnose erstellt wurde, auf die Steroide an. Und drittens wurde in dem Material, das bei der ersten Bronchoskopie vor Verabreichung von PSL gewonnen wurde, Cryptococcus nicht nachgewiesen.

Der Patient verwendete in seinem landwirtschaftlichen Betrieb täglich auf der Basis von Hühnerkot hergestellte Düngemittel. Daher war er Tag für Tag gegenüber Cryptococcus exponiert und infizierte sich so - was nicht überrascht - während der Steroidtherapie mit Cryptococcus. Es besteht dennoch auch die Möglichkeit, dass er bereits bei Auftreten des ersten Symptoms mit Cryptococcus infiziert war, da bei ihm vor Verabreichung des Kortikosteroids keine Untersuchung auf Kryptokokken-Antigen im Serum erfolgte.

Zusammengefasst kann eine Lungenkryptokokkose bei immunkompetenten und immungeschwächten Patienten zu multiplen Konsolidierungen im Thorax-CT führen. Ungeachtet ihrer Seltenheit sollte bei Patienten mit COP, die auf eine Steroidbehandlung nicht ansprechen, eine zugrunde liegende Lungenkryptokokkose in Betracht gezogen werden. Dies gilt insbesondere in Fällen, in denen nach initialer Besserung durch die Steroidbehandlung eine Verschlechterung beobachtet wird.

\section{Disclosure Statement}

Von dem Patienten wurde nach Aufklärung eine schriftliche Einverständniserklärung für die Veröffentlichung des vorliegenden Fallberichts und der dazugehörigen Bilder eingeholt.

\section{Literatur}

Die Literatur ist unter https://www.karger.com/Article/Fulltext/510568 abrufbar.

\section{Lizenzangabe}

Motoko Nomura, Hiromitsu Ohta, Masahiro Hirutaet al.: Steroid resistance in organizing pneumonia caused by pulmonary cryptococcosis. Respirol Case Rep. 2020;8(4):e00556. (c) 2020 The Author(s) Respirology Case Reports published by John Wiley \& Sons Australia, Ltd on behalf of The Asian Pacific Society of Respirology. (Übersetzung), lizensiert unter CC BY 4.0 (https://creativecommons.org/licenses/by/4.0/deed.de). 